\title{
Tracking keystroke sequences at the cortical level reveals the dynamics of serial order production
}

\author{
Svetlana Pinet ${ }^{1,2}$, Gary S. Dell ${ }^{3}$, F.-Xavier Alario ${ }^{1}$ \\ 1. Aix Marseille Univ, CNRS, LPC, Marseille, France \\ 2. Department of Neurology, Johns Hopkins University, 1629 Thames Street, Suite 350, \\ Baltimore, MD 21231, USA \\ 3. University of Illinois, Department of Psychology, Champaign, IL 61820 \\ USA
}

\begin{abstract}
Response selection is often studied by examining single responses, although most actions are performed within an overarching sequence. Understanding processes that order and execute items in a sequence is thus essential to give a complete picture of response selection. In this study, we investigate response selection by comparing single responses and response sequences, as well as unimanual and bimanual sequences. We recorded EEG while participants were typing one- or two-keystroke sequences. Irrespective of stimulus modality (visual or auditory), response-locked analysis revealed distinct contralateral and ipsilateral components previously associated with activation and inhibition of alternative responses. Unimanual sequences exhibited a similar activation/inhibition pattern as single responses, but with the activation component of the pattern expressed more strongly, reflecting the fact that the hand will be used for two strokes. In contrast, bimanual sequences were associated with successive activation of each of the corresponding motor cortices controlling each keystroke, and no traceable inhibitory component. In short, the activation component of the two-keystroke sequence EEG pattern can be understood from the addition of activation components of single-stroke sequences; the inhibition of the hand not being used is only evidenced when that hand is not planned for the next stroke.
\end{abstract}

Keywords: Competitive Queuing, Electroencephalography, Inhibition, Motor Planning, Language

Corresponding author:

Svetlana Pinet

Department of Neurology, School of Medicine, Johns Hopkins University

1629 Thames Street, Suite 350,

Baltimore, MD 21231, USA

spinet1@jhmi.edu 


\section{Introduction}

Producing a behavioral sequence involves retrieving and activating each sequence unit in an ordered and timely fashion, a requirement often referred to as the problem of serial order (Lashley, 1951). An important theory of serial order is expressed by Competitive Queuing (CQ) models. These models have been successfully applied in several cognitive domains, such as memory (e.g., serial recall, Houghton, 1990) and language (e.g., typing, Rumelhart and Norman, 1982). CQ models propose that all elements of a sequence are activated, such that there is a gradient of activation consistent with the order of items in the sequence. For example, the gradient may be set up by each item inhibiting all subsequent items, and the sequence may be executed by selecting the currently most activated item, and then inhibiting it, thus leading to the next item in the queue possessing the most activation. Then, a balance of activation and inhibition of items leads to the correct production of the planned sequence.

The CQ model's activation and inhibition processes constitute an algorithmic (Marr, 1982) account, one that makes no claims about physiological implementation. A significant step toward such an implementation was taken by Averbeck et al. (2002), who showed that the prefrontal cortex of behaving monkeys displayed a gradient of activation of future items, their degree of activation following their order in the sequence. These data show that at least some assumptions from cognitive models of sequence planning can be linked to physiological activities, and hence to their neural implementation.

In the case of humans, when they give manual responses with either hand it is possible to follow the activation of a response and the inhibition of its alternative with electroencephalographic (EEG) recordings (e.g., Servant et al. 2016). Readiness potentials and their lateralized computation (LRP) have been widely used to describe such dynamics (Coles, 1989; de Jong, Wierda, Mulder, \& Mulder, 1988; Gratton, Coles, Sirevaag, Eriksen, \& Donchin, 1988). However, a more detailed approach consists of using a spatial filter (Laplacian transform) that allows more direct assessment of the activity of each motor cortex (Perrin, Pernier, Bertrand, \& Echallier, 1989; Vidal et al., 2015). Using this approach, the component recorded over the contralateral motor cortex of a response effector has been directly linked to the activation of that cortical area, and the analogous ipsilateral component has been linked to the inhibition of the corresponding ipsilateral area (Burle, Vidal, Tandonnet, \& Hasbroucq, 2004; Donner, Siegel, Fries, \& Engel, 2009; Selen, Shadlen, \& Wolpert, 2012; Vidal, Grapperon, \& Bonnet, 2003).

What this research on single responses does not address is whether and how these components and processes would be involved in sequence production, minimally a sequence of two strokes on a keyboard. The simplest additive hypothesis would state that the production of two responses can be understood from the addition of each single response. Figure 1 presents the predictions of such a hypothesis on the associated motor components, separately for unimanual (both responses use the same hand) and bimanual (each response uses a different hand) sequences, based on the components known to be associated with single responses. The additive hypothesis was schematized by considering that a two-stroke sequence consists of the simple addition of each component from the single stroke, adjusted for the side of each keystroke, and with a 170ms delay between keystrokes (estimated from the recorded average inter-keystroke interval). For unimanual sequences, the activation and inhibition components associated with each keystroke are added and each hemisphere would then exhibit something like double the amplitude compared to the components associated with single responses. Bimanual sequences require the inversion of the role of each hemisphere in terms of activation and inhibition from the first to the second keystroke. The addition of the resulting 
components yields specific patterns for each hemisphere, each of which is quite different from the expectation for unimanual sequences. Any deviation from the expected patterns displayed in Figure 1 would indicate the existence of specific sequence mechanisms.
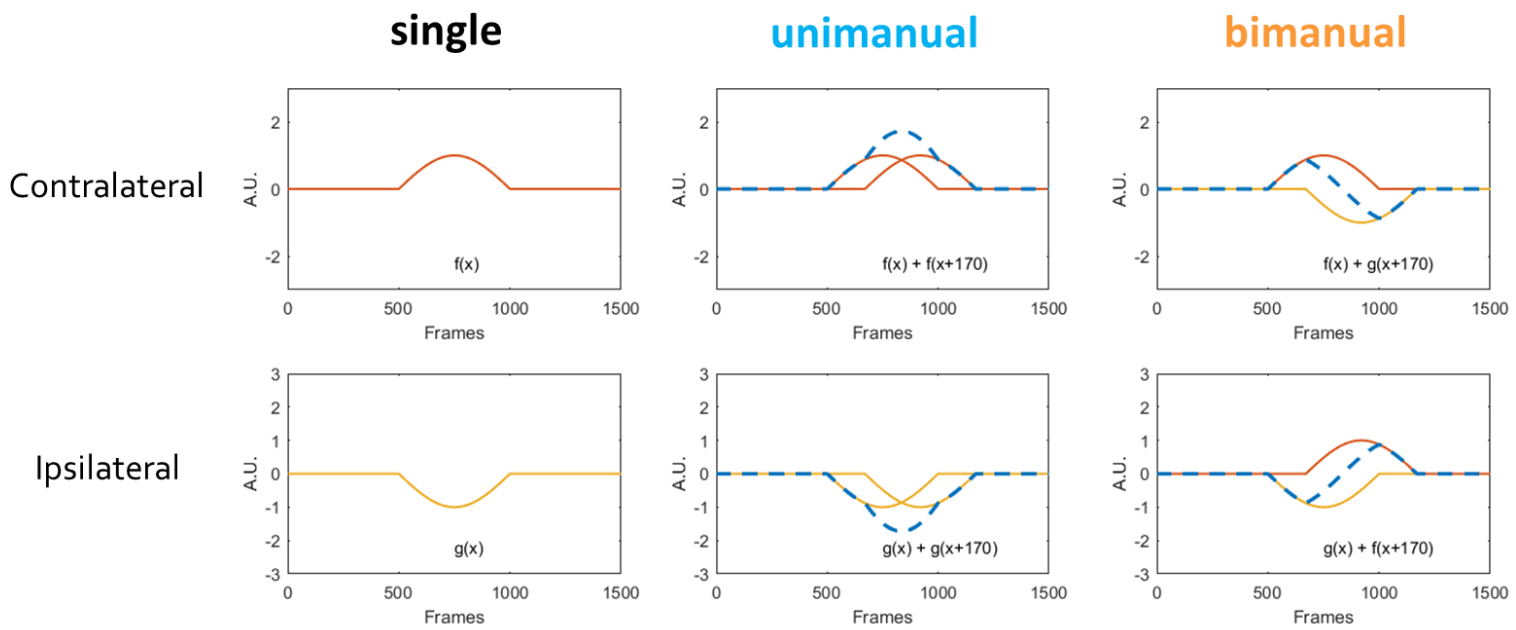

Figure 1. Schematic illustration of the additive hypothesis. "Electrodes" contralateral and ipsilateral to the first keystroke are plotted separately. An orange line represents the contralateral component ' $\mathrm{f}(\mathrm{x})$ ' and a yellow line represents the ipsilateral component ' $\mathrm{g}(\mathrm{x})$ '. A dashed blue line corresponds to the addition of the components for each electrode, and represents the components predicted by the additive hypothesis (see main text for details). Equations on each graph specify the addition of components that was computed. The second keystroke is modeled to be delayed of $170 \mathrm{~ms}$ relative to the first keystroke.

Previous studies about bimanual sequence production are not incompatible with the above predictions. Focusing on the preparation period before an execution cue, the LRP can index the amount of preparation of the following sequence. The LRP displays a higher amplitude when two movements are being prepared rather than one (de Jong, Gladwin, \& M't Hart, 2006; Gladwin, 't Hart, \& de Jong, 2008). However, results also show that bimanual movements cannot be easily understood as the sum of two unimanual movements as evidenced in particular on the motor potentials, suggesting that specific processes might be in place during sequence preparation (R. Q. Cui \& Deecke, 1999). In particular, the effectors involved in a movement sequence influence how the sequence is being prepared, initiated and executed. However, without separating activity coming from each hemisphere, it is not possible to formulate precise predictions, especially those regarding the inhibition (ipsilateral) component, and thus linking neurophysiological results with the hypothesis of cognitive models.

In contrast with evoked potentials, time-frequency estimates reveal the selection of motor goals, or the foreknowledge of a sequence (Gladwin et al., 2008; Park, Kim, \& Chung, 2013). Both alpha- and beta-band show desynchronization at the start of a movement sequence, sustained for the whole duration of the movement (Alegre et al., 2004). Due to the slower time-frame of processes indexed by time-frequency, however, potentials seem more appropriate in order to test the predictions laid out by the additive model, as they reflect processes closer to response initiation, of particular interest here (Gladwin et al., 2008).

The activation and inhibition components described above for single responses have been shown to be present in the context of word typing (Pinet et al. 2015; Pinet et al. 2016; Scaltritti et al. 2017; see also Logan et al. 2011), a quintessential serial order task that could provide a simple and natural domain for addressing sequence production. Most recently, Scaltritti et al. (2018) explored the scope of planning in typing. They showed that the neurophysiological signature associated with response selection is 
modulated by item position within the upcoming sequence (close vs. distant). Consistently, Behmer et al. (2018) used trans-cranial magnetic stimulation (TMS) to show a gradient of activation of motor cortex that corresponded to the graded position of future keystrokes.

The goal of our work was to identify the physiological implementation of some assumptions made by cognitive models, such as the CQ model, and particularly the assumptions regarding the successive activation of each item, and the inhibition of upcoming items. In the present study, we tracked the cortical dynamics driving response programming in single keystrokes and two-keystroke sequences that were either unimanual or bimanual. Because previous studies allowed us to formulate precise predictions on activation/inhibition components (Figure 1), we restricted our analysis to the time domain. Comparing single keystrokes to two-keystroke sequences informs us about whether and when activation and inhibition processes identified for single responses are modified by the requirement to type an additional keystroke in a sequence context. Each contrast of sequence type (unimanual or bimanual) vs. single strokes informs us further about the interplay between the hemispheres. Specifically, the unimanual vs. single contrast keeps the recruited effectors constant but increases the number of strokes in the unimanual case, perhaps requiring more processing from the contralateral hemisphere. The bimanual vs. single contrast tests the role of both effectors, particularly the possible influence of inhibition of the hemisphere not involved in the immediate keystroke.

\section{Methods}

\section{Participants}

Eighteen right-handed participants were selected for their ability to touch-type, i.e. typing with all (or almost all) 10 fingers without need for looking at their hands. Four participants were excluded because of problematic eye movements around the moment of response or poor signal-to-noise ratio. The final sample (5 males, 9 females) was 24.9 years old on average (range: 20-33 years old). Participants' typing ability was assessed via a typing test (e.g. Pinet et al. 2016). Participants had a mean accuracy of $84 \%$ (range $78-91 \%$ ) and a mean typing speed of $48 \mathrm{wpm}$ (5-character words per minute; range 34.6-57.5 wpm).

\section{Stimuli and design}

Eight single letters and six two-letter words were used as stimuli. Half of the single letters were typed with the left hand ("Z", "E", "S", "D") and the other half with the right hand ("I", "O", "K", "L") in the AZERTY keyboard. The words selected were the French names of musical notes, to ensure homogeneity of meaning, and because they present interesting sequence properties. Four musical notes names started with the left hand ("DO", "RE", "FA", "SI") and two with the right hand ("MI", "LA"); half were typed with one hand (unimanual: "RE", "FA", "MI") and the other half with both hands (bimanual: "DO", "SI", "LA"). Hence, stimuli were divided into three conditions: single (one letter), unimanual (two letters, one hand), bimanual (two letters, two hands). The only three letter musical note ("SOL") was also included for completeness, but data from SOL trials was not analyzed. Stimulus presentation was visual (printed letters) and auditory (spoken letter or music note names). Stimulus modality (visual vs. auditory) and stimulus type (letters vs. musical notes) were presented in four independent blocks. Each participant saw all conditions in a randomized order.

\section{Procedure}

Visual stimuli were displayed on a 17 -inch CRT computer screen placed at about $70 \mathrm{~cm}$ from the participant. Auditory stimuli were presented binaurally through earphones. Responses were collected 
from a DirectIN High Speed Keyboard PCB v2010 manufactured by Empirisoft to get keystroke timing data with at least $1 \mathrm{~ms}$ accuracy. Stimuli presentation and response acquisition were controlled using the software Presentation (NeuroBehavioral Systems).

Participants sat in a dimly lighted electrically shielded room. Their seating position was adjusted so that they were comfortable typing. The task was to type the letter or musical note name on the keyboard as fast and as accurately as possible after presentation. "Letter blocks" comprised 200 trials (25 repetitions of each of the eight stimuli). "Music blocks" comprised 350 trials (50 repetitions of each of the seven note names). Each block comprised a short break.

A trial consisted of a fixation cross presented during a random duration from $400 \mathrm{~ms}$ to $600 \mathrm{~ms}$, followed by presentation of the stimulus (maximal duration $2000 \mathrm{~ms}$ ). After the response, the stimulus stayed on for $500 \mathrm{~ms}$, then feedback (correct or incorrect, indicated by a green check mark or a red cross or high and low pitch beeps) was displayed for $500 \mathrm{~ms}$ in the same modality as the stimulus (visual or auditory). The screen then remained black for $500 \mathrm{~ms}$. Every two to three trials, there was a 2 s interval to allow participants to blink and avoid contamination of the signal.

\section{Behavioral data analysis}

Response times (RT, time of the first keystroke) and accuracy rates were analyzed with linear mixedeffect models, as is commonly done in cognitive studies of language (Baayen, Davidson, \& Bates, 2008; Jaeger, 2008). RTs were log-transformed, following the result of a Box Cox test. RTs below 300 $\mathrm{ms}$ or incorrect responses were removed from the RT analysis. RTs and accuracy rates were analyzed according to the following predictors: Modality (Auditory or Visual), 1st keystroke hand (left or right), 2nd keystroke (single, unimanual, bimanual). To account for the 3 levels of the 2nd keystroke predictor, the predictors were Helmert-coded to contrast sequence length (one- vs. two- keys) and $2^{\text {nd }}$ keystroke side (same/different). By-participant random slopes were added for all predictors of interest. Random intercepts by-participant and by-item were also added to the model. Trial number was added as a control variable. Accuracy rate analysis included a random slope for Modality only, as the model would not converge otherwise. Since the computation of p-values in this kind of analysis is debated, we considered t-values to approximate $\mathrm{z}$ values and considered "significant" any value above 1.96 (Bates, Mächler, Bolker, \& Walker, 2015).

\section{EEG recordings and preprocessing}

EEG was acquired from 128 scalp locations using $\mathrm{Ag} / \mathrm{AgCl}$ active electrodes (BioSemi Active Two system), referenced to the CMS-DRL ground. The sampling rate was $512 \mathrm{~Hz}$ (filters: DC to $104 \mathrm{~Hz}, 3$ db/octave slope). Vertical (VEOG) and horizontal (HEOG) electrooculograms were recorded with three surface electrodes $(\mathrm{Ag} / \mathrm{AgCl})$ placed below the left eye and next to the two outer canthi.

Offline analyses were performed using the MATLAB toolboxes Brainstorm (Tadel, Baillet, Mosher, Pantazis, \& Leahy, 2011), and Fieldtrip (Oostenveld, Fries, Maris, \& Schoffelen, 2011), as well as custom routines. Data were re-referenced to the average of both mastoids. Continuous data were filtered (Butterworth Filter Order 4: 0.1-100 Hz). Bad electrodes were removed and interpolated. A first mild artifact rejection was performed before computing ICA (algorithm: Infomax from EEGLAB toolbox, Delorme and Makeig 2004). Component(s) corresponding to eye movements was (were) removed. A second stricter artifact rejection was then performed to reject remaining noisy segments. A -200 to $0 \mathrm{~ms}$ pre-stimulus baseline was applied by subtraction. Response-locked epochs were then extracted (-1500 ms : $+800 \mathrm{~ms}$ around first or second keystroke).

Current source density transformation was applied (number of spherical splines 4; maximal degree of Legendre polynomial: 20, smoothing parameter: 10-5) to enhance spatial resolution of the EEG signal (Perrin et al., 1989). 


\section{EEG statistical analysis}

Unless stated otherwise, activity on the contralateral hemisphere from left- and right-hand responses were averaged together. Similarly, activity on ipsilateral hemisphere from left- and right-hand responses were averaged together. The signal is presented relative to left hand responses (i.e., ipsilateral hemisphere corresponds to left hemisphere).

Two main contrasts (unimanual vs. single and bimanual vs. single) were tested from $-500 \mathrm{~ms}$ to 0 including all 128 electrodes, using cluster based non-parametric statistics (Maris \& Oostenveld, 2007). This procedure allows us to deal with the multiple comparison problem by clustering significant neighboring channels and time-points and building the statistical distribution directly from the observed data by successive permutations. More specifically, two conditions are compared with a paired t-test at each time-point. Clusters are composed of the time-points presenting t-values above a specific threshold $(\mathrm{p}<.05)$ based on spatial and temporal adjacency. The sum of $\mathrm{t}$-values within each cluster gives the cluster-level statistic, and the significance of each cluster is computed using permutations. However, one should refrain from over-interpreting the spatial or temporal extension of any cluster. Because the significance is only tested between conditions, one should not conclude strongly about the boundaries of any cluster (any point is not tested against another). Nevertheless, we depict the "significant clusters" as channel x time matrices for illustrative purposes. Although all electrodes within a cluster display a significant effect, there exists some variability of the pattern each electrode display. For that reason, we display results over an array of significant electrodes rather than the averaged signal over cluster electrodes. In order to compare our results to previous literature, we especially display specific electrodes over the motor cortex, close to $\mathrm{C} 3 / \mathrm{C} 4$ (i.e. D18 and B21 in our 128-electrode system), based on Pinet et al. (2016).

The two main contrasts were run for data collapsed over modality and hand (main analysis reported). They were also run for each modality and hand separately ( 8 additional contrasts).

\section{Results}

\section{Behavioral results}

The RT analysis (see Table 1) revealed a main effect of Modality, with auditory stimuli eliciting slower responses than visual stimuli $\left(\beta=0.14, \mathrm{t}=4.5, \mathrm{CI}=[0.076 ; 0.20], \mathrm{M}_{\text {visual }}=697.7 \pm 231.4 \mathrm{~ms}\right.$, $\mathrm{M}_{\text {auditory }}=792.4 \pm 255.3 \mathrm{~ms}$ ). Response times also exhibited a practice effect, strongly decreasing with trial number $(\beta=-8.43 \mathrm{E}-5, \mathrm{t}=-13.2, \mathrm{CI}=[-9.7 \mathrm{E}-5 ;-7.2 \mathrm{E}-5])$. None of the other effects was significant.

Mean accuracy was $93.5 \%$. Accuracy rates analysis (see Table 2) revealed that unimanual sequences were produced more accurately than bimanual sequences $\left(B=1.1, \mathrm{z}=3.4, \mathrm{CI}=[0.45 ; 1.7], \mathrm{M}_{\text {unimanual }}=\right.$ $\left.94.1 \pm 2.8 \%, \mathrm{M}_{\text {bimanual }}=93.1 \pm 5.0 \%\right)$. None of the other effects was significant.

Table 1. Mixed model regression results for RT.

\begin{tabular}{|l|l|l|l|l|l|l|}
\hline Fixed effects & Coefficient & SE & t & \multicolumn{1}{l|}{ CI (95\%) } & p \\
\hline Intercept & 6.568 & 0.046 & 143.115 & 6.478 & 6.658 & $<2 \mathrm{e}-16$ \\
\hline Modality (Visual/Auditory) & 0.136 & 0.031 & 4.46 & 0.076 & 0.196 & $6.49 \mathrm{E}-04$ \\
\hline First Key (left/right) & -0.051 & 0.029 & -1.78 & -0.108 & 0.005 & 0.090 \\
\hline Second Key (one-/two-keys) & 0.036 & 0.035 & 1.04 & -0.032 & 0.104 & 0.312 \\
\hline Second Key (same/different hand) & -0.012 & 0.043 & -0.28 & -0.097 & 0.073 & 0.782 \\
\hline Trial & $-8.43 \mathrm{E}-05$ & 0.000 & -13.22 & $-9.68 \mathrm{E}-05$ & $-7.18 \mathrm{E}-05$ & $<2 \mathrm{e}-16$ \\
\hline & & & & & & \\
\hline
\end{tabular}




\begin{tabular}{|l|l|l|l|l|l|l|}
\hline Random effects & Variance & & & & & \\
\hline Subject Intercept & 0.027 & & & & & \\
\hline Modality |Subject & 0.013 & & & & & \\
\hline First Key | Subject & 0.006 & & & & & \\
\hline Second Key (one-/two-keys) | Subject & 0.011 & & & & & \\
\hline Second Key (same/different hand) | Subject & 0.015 & & & & & \\
\hline Item Intercept & 0.001 & & & & & \\
\hline Residual & 0.046 & & & & & \\
\hline
\end{tabular}

Table 2. Mixed model logistic regression results for accuracy rates.

\begin{tabular}{|l|l|l|l|l|l|l|}
\hline Fixed effects & Coefficient & SE & t & CI (95\%) & p \\
\hline Intercept & 2.937 & 0.229 & 12.813 & 2.488 & 3.386 & $<2 \mathrm{e}-16$ \\
\hline First Key (left/right) & -0.044 & 0.212 & -0.209 & -0.459 & 0.371 & 0.834 \\
\hline Second Key (absent/present) & 0.037 & 0.210 & 0.177 & -0.375 & 0.450 & 0.859 \\
\hline Second Key (same/different hand) & 1.057 & 0.308 & 3.432 & 0.453 & 1.661 & $6.00 \mathrm{E}-04$ \\
\hline Modality (Visual/Auditory) & 0.078 & 0.117 & 0.667 & -0.152 & 0.308 & 0.505 \\
\hline & & & & & & \\
\hline Random effects & Variance & & & & & \\
\hline Subject Intercept & 0.433 & & & & & \\
\hline Modality |Subject & 0.100 & & & & & \\
\hline Item Intercept & 0.127 & & & & & \\
\hline
\end{tabular}

\section{The signature of sequence programming depends on its constituents}

EEG analyses were performed on correct trials only. Trials presenting EEG artifacts (19.4\%) were rejected. Response-locked averages are depicted on Figure 2. All topographies are presented relative to left hand responses (i.e., right hemisphere corresponds to contralateral hemisphere). We depict one representative electrode on each side (contralateral (B21) and ipsilateral (D18) to the response, near $\mathrm{C} 3$ and $\mathrm{C} 4$ in the standard 10-10 system), to provide comparison with previous studies (e.g., Vidal et al. 2003; Pinet et al. 2016).

Prior to the execution of a single keystroke (single condition, Figure 2a, left), we observed a clear negativity/positivity pattern over contralateral/ipsilateral hemispheres, replicating the pattern repeatedly observed in 2AFC tasks (Meynier, Burle, Possamaï, \& Vidal, 2009; Vidal et al., 2003) and more recently in word typing (Pinet et al., 2016, 2015, Scaltritti et al., 2017, 2018). The same general pattern is observed for unimanual sequences (Figure 2b). In contrast, both the topographies and the ERP time courses indicate a different pattern for bimanual sequences, with a negative central component present on both ipsilateral and contralateral hemispheres, and a symmetric topography. 
a

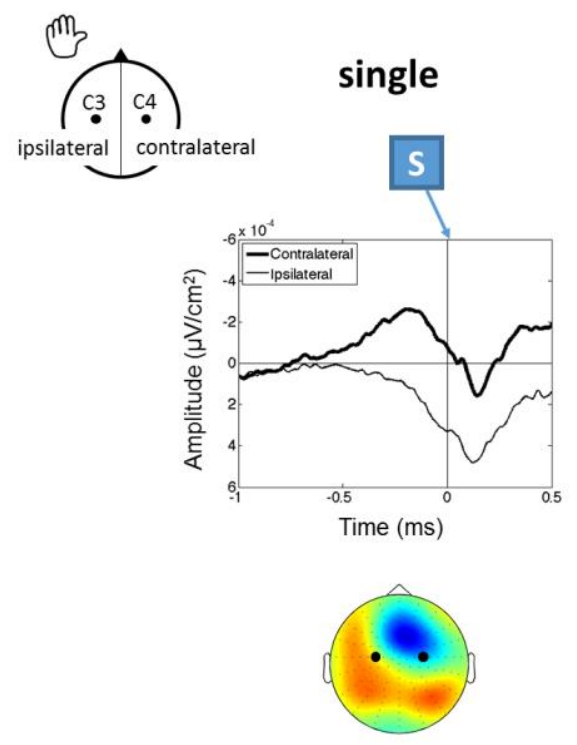

b

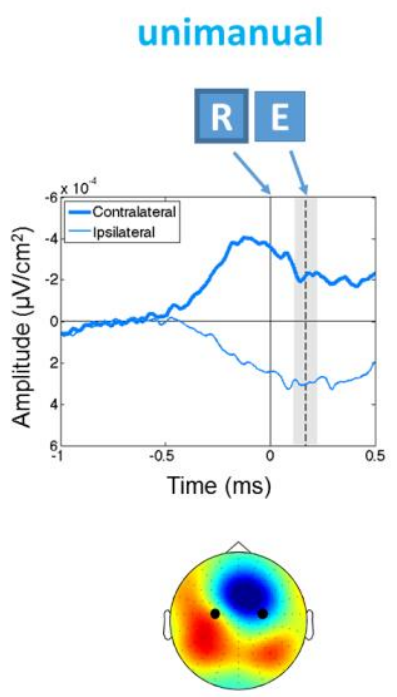

C

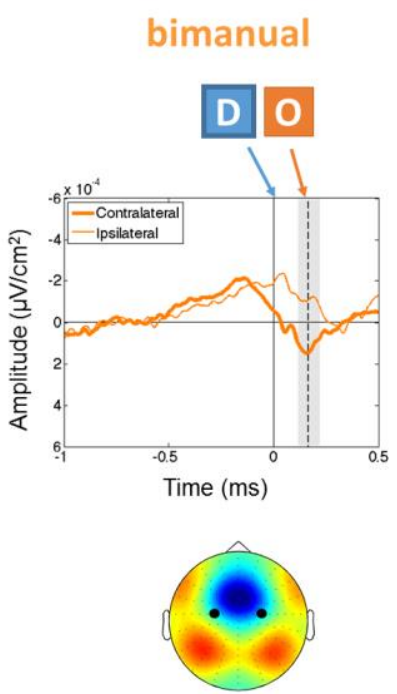

Figure 2. Activities recorded over contralateral and ipsilateral hemispheres for single, unimanual and bimanual conditions. Zero represents the first keystroke. The dotted line represents the mean time interval between keystrokes in two-keystroke conditions (shaded area: standard deviation). Topographies are plotted in the [-200:$150 \mathrm{~ms}$ ] time-window, corresponding to latency of the contralateral peak, and displayed contralateral and ipsilateral electrodes are indicated by black dots on the topographies.

Direct comparisons between conditions over a range of electrodes are presented on Figure 3a. Unimanual sequences (vs. single, see Figure 3a) yielded an amplitude increase of the negative component mainly over contralateral hemisphere (cluster-based non-parametric test: $p=0.016$, Figure $3 \mathrm{~b}$ ), but virtually no modulation of the ipsilateral positivity. The topography of the statistic confirmed an effect focused over central and contralateral electrodes.

Bimanual sequences contrasted with single responses in a different way. Focusing first on central electrodes, bimanual sequences (vs. single, see Figure 3a) presented a difference over ipsilateral hemisphere (cluster based non-parametric test: $p=0.002$, Figure 3c). The ipsilateral component in bimanual sequences was of opposite polarity than in single responses, clearly suggesting different processes in the contra- and ipsilateral hemispheres. The topography of the statistic confirmed that this positive effect was focused on midline and ipsilateral electrodes. Second, peripheral contralateral electrodes also presented a negative difference in amplitude (cluster based non-parametric test: $\mathrm{p}=$ 0.02). This stems from the fact that central components were more spatially focused in the bimanual condition (see topography in Figure 2c, and Figure 3) compared to the single condition, which could indicate a mixture of processes in the production of bimanual sequences (see Discussion below).

These two contrasts reveal that the contralateral and ipsilateral components are modulated by different factors, hence stem from distinct processes.

Over midline frontal electrodes (see Figure 3a), higher amplitude was observed for both uni- and bimanual sequences relative to single (confirmed by cluster based non-parametric tests reported above, midline electrodes being part of the positive clusters). Thus, midline electrodes presented a very different pattern from their neighboring lateralized electrodes.

The patterns described were observed in each stimulus modality (visual or auditory) and were obtained regardless of the hand of the first (or only) keystroke (i.e., without mirror averaging). Only some of 
eight contrasts tested, however, reached significance when the data were broken down by hand or modality, presumably because of lack of power.

a.
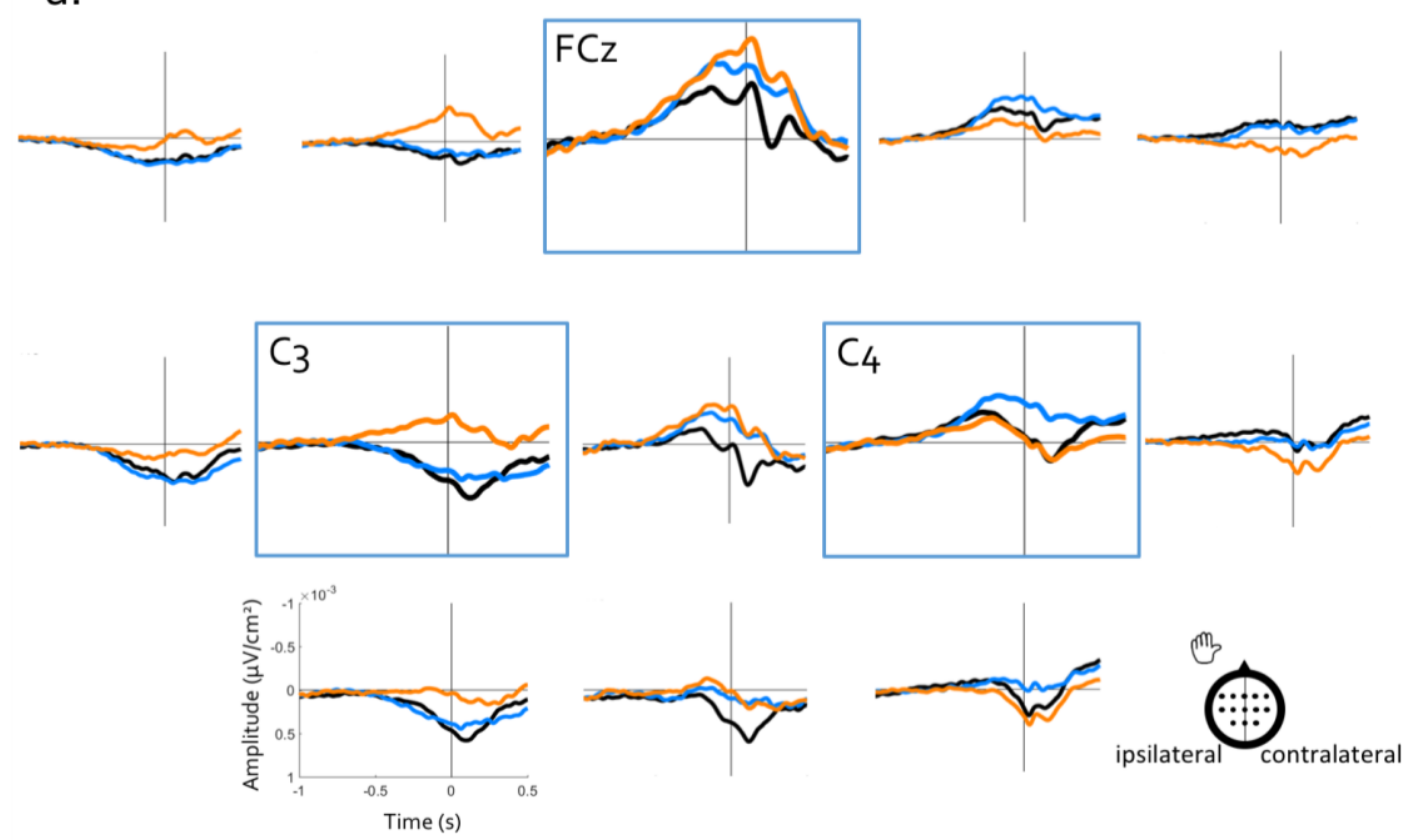

b. unimanual vs. single

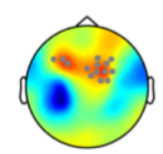

$-300 \mathrm{~ms} \quad-200 \mathrm{~ms}$

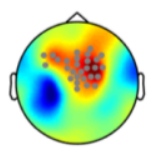

$-100 \mathrm{~ms}$

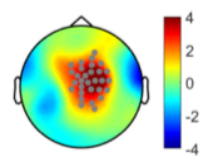

o ms c. bimanual vs. single

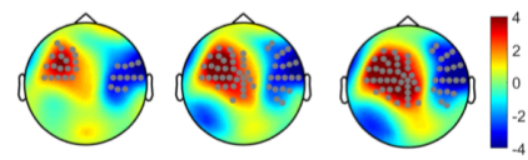

$-300 \mathrm{~ms} \quad-200 \mathrm{~ms} \quad-100 \mathrm{~ms} \quad$ oms
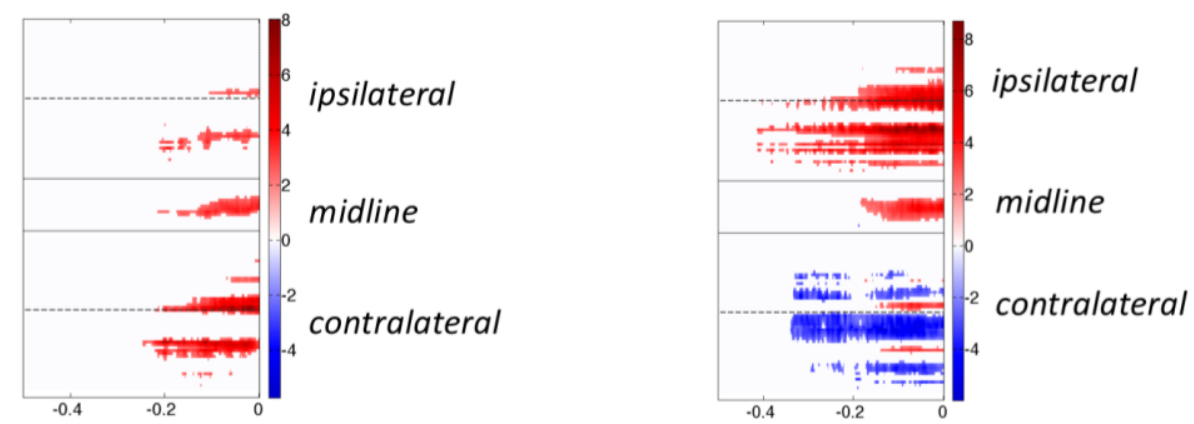

Figure 3. Evoked potentials results. (a) Response-locked potentials comparing unimanual (blue) and bimanual (orange) conditions against single (black). The position of the 13 electrodes displayed is indicated on the scalp. $\mathrm{C} 3, \mathrm{C} 4$ and $\mathrm{FCz}$ electrodes are displayed larger. (b, c) Results of the cluster-based permutation tests for unimanual vs. single (b) and bimanual vs. single (c) contrasts. Results are presented as topographies from -300 $\mathrm{ms}$ to $0 \mathrm{~ms}$ in $100 \mathrm{~ms}$ time-windows, with significant electrodes in each time-window outlined as grey dots. A matrix of channels x time-frames, grouped by ipsilateral hemisphere, midline and contralateral hemisphere, ordered from posterior to anterior, masked for statistical significance indicates the full extent of the cluster in space and time. Zero represents the first keystroke. 


\section{Preparation of a bimanual sequence}

A closer look at bimanual sequences is informative about timing. The data from these sequences differed considerably from the pattern obtained in the single and unimanual conditions. Instead of the expected positive component, the ipsilateral hemisphere presented a negative component whose timecourse was similar to the contralateral component, albeit shifted in time (Figure 2c). We extracted the peak latency for each component on the grand-averaged ERPs on contralateral (B21) and ipsilateral (D18) electrodes in the $[-500 \mathrm{~ms}:+200 \mathrm{~ms}]$ time-window around the first keystroke. This temporal shift measured on average ERPs between ipsilateral and contralateral peaks (184 ms) was in the range of the average inter-keystroke interval (mean $=167 \pm 50 \mathrm{~ms}$ ). Extracting timing from averaged ERP waves is not that straightforward ("the average of the mean is not the mean of the average", Fabiani et al. 1987; Meyer et al. 1988). Still, the timing difference observed between the two peaks is compatible with the interpretation of the second peak as being linked to the second stroke. In contrast, in the unimanual condition only one peak was visible, over the contralateral hemisphere (Figure 2b), while the mean inter-keystroke interval was in the same range (mean $=171 \pm 51 \mathrm{~ms}$ ).

We also examined each bimanual sequence separately (left-right or right-left keystroke sequences; Figure 4a). Peak latencies were extracted for each subject on contralateral (B21) and ipsilateral (D18) electrodes in the $[-500 \mathrm{~ms}:+200 \mathrm{~ms}]$ time-window around the first keystroke. In both sequence types, the component recorded over the hemisphere contralateral to the first keystroke always peaked before the component over the ipsilateral hemisphere, as confirmed by a Wilcoxon signed-rank test (LR sequences: $z=-2.48, p=.013$; RL sequences: $z=-2.61, p=.009$ ), despite the signal being noisier than in the main analysis. That is, the order of peak activity across hemispheres followed the order of the keystrokes performed.

In addition, the contralateral components considered relative to the first or second keystroke were quite similar, irrespective of the upcoming keystroke (Figure 4b). Notably, the timing or relative position of each component relative to the corresponding keystroke was similar.

Together, the above observations consistently converge to indicate that the ipsilateral peak is indeed associated with the preparation of the second keystroke. 
a

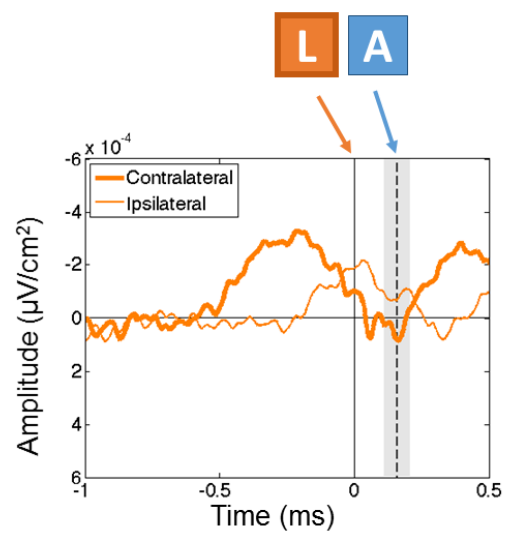

b

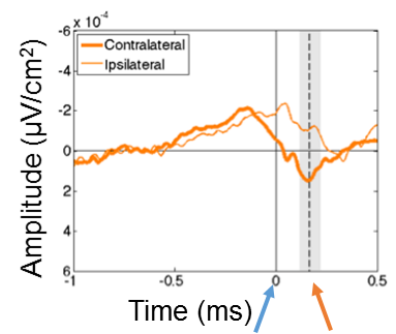

D 0

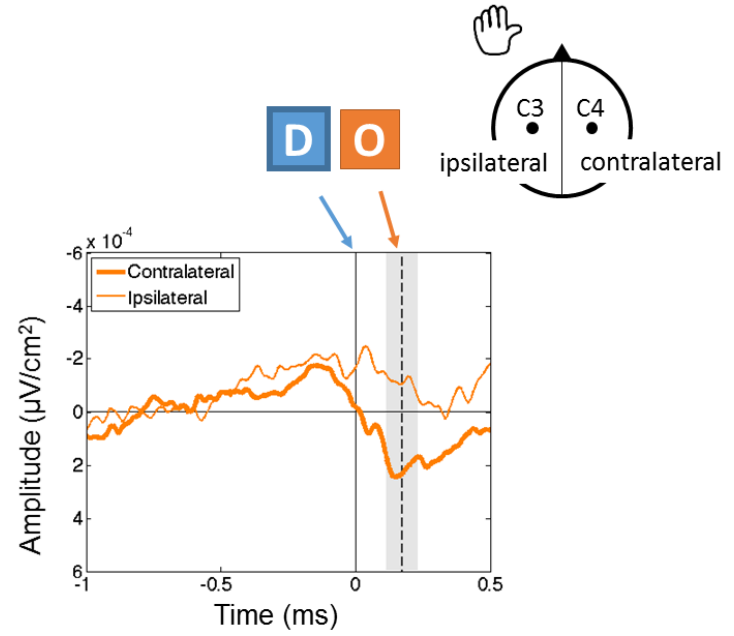

D 0

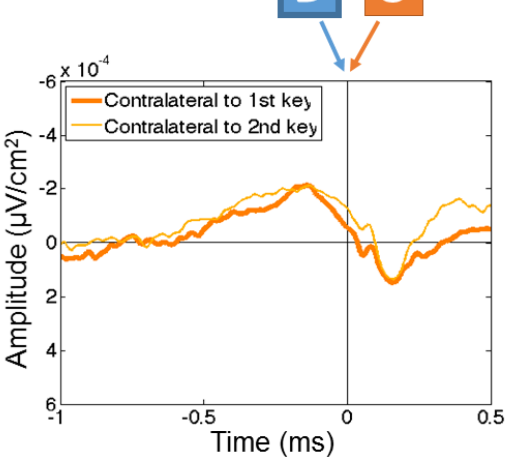

Figure 4. Response-locked averages in the bimanual condition. (a) Bimanual sequences that start with a left keystroke (LEFT-right, e.g., "DO") or a right keystroke (RIGHT-left, e.g., "LA") are presented separately. Contralateral and ipsilateral hemisphere are defined relative to the first keystroke of each sequence. Zero represents the first keystroke. (b) In order to track preparation of each keystroke, signal is plotted over the hemisphere contralateral to the forthcoming keystroke. Zero represents the first or second keystroke accordingly. Small figure on the left is similar to Figure $2 \mathrm{c}$ and is provided to indicate time stamps relative to each keystroke along execution of the sequence. 
Keystroke sequences at the cortical level

\section{Discussion}

In this study, we tested hypotheses derived from competitive queuing models (CQ) models of sequence production using neurophysiological indices. In particular, we sought to understand how activation and inhibition processes are employed during the production of a sequence. Our starting point was the programming of a single keystroke (single condition). This condition yielded the expected pre-response pattern of a negative and a positive component (e.g., Vidal et al. 2003) that has been consistently interpreted in the cognitive control literature as, respectively, contralateral activation and ipsilateral inhibition (Burle et al., 2004; Diedrichsen, Wiestler, \& Krakauer, 2013; Meckler et al., 2011; Servant et al., 2016). Our single-key results go further than previous single-response studies, however, in that our results were obtained with eight alternative responses (the eight letters). Previous studies contrasted fewer responses (up to four: Meynier et al. 2009).

We then manipulated the number of keystrokes to be produced (up to two) and the effectors used to produce them (one or both hands). Thereafter, any deviation from the single pattern will necessarily be explained by the presence of a second keystroke. To ease the interpretation of our results, we schematically illustrate the observed patterns in Figure 5 and compare them to the predictions made in the Introduction (Figure 1, reproduced in Figure 5a). This schematized description was inspired by Figure 4 in Greenhouse et al. (2015), among others. A decomposition of the observed pattern as the "single" patterns that would be associated with each keystroke in the sequence is presented in Figure $5 \mathrm{c}$ and will guide our interpretation of the results.

Activity over the contralateral hemisphere is informative about the dynamics of keystroke activation. In the single and bimanual conditions, a component of similar amplitude is observed over the contralateral hemisphere associated with the production of a single keystroke. This suggests that similar processes are associated with the activation of each single keystroke, regardless of whether this keystroke is part of a sequence or isolated. In line with this idea, in a bimanual sequence, each keystroke is associated with such a component over the hemisphere contralateral to each respective keystroke; in a unimanual sequence, the hemisphere contralateral to both keystrokes presents a component of around twice the amplitude than in single, in line with the additive hypothesis (see Figure 5b). Both these results confirm the idea that activation of a keystroke is an additive process: preparing one right-handed response leads to a left-side brain response, and preparing two such responses, leads to a doubly-strong left side response (R. Q. Cui \& Deecke, 1999; Gladwin et al., 2008).

Beyond the electrodes of primary interest, the spatial extent of the components was less distributed in bimanual sequences than in single and unimanual conditions, thus yielding a significant contrast over peripheral electrodes. This result may be indicative of a mixture of processes in the bimanual condition, even though our data cannot provide definitive evidence in that respect (R. Q. Cui \& Deecke, 1999; Diedrichsen et al., 2013)

In addition, activation closely follows the sequence of keystrokes. This is especially visible in the bimanual condition, where sequential activation can be traced over opposite hemispheres following the order of keystrokes (Figure $5 \mathrm{c}$ ). There, the time between activation peaks corresponds to the average interkeystroke interval. This is evidence for an electrophysiological pattern that mirrors the sequential launching of each item in the sequence.

Overall, then, contralateral neurophysiological activity associated with response activation follows what was expected from single response data, namely additive response activations (Gladwin et al., 2008). 


\section{a. Additive hypothesis}
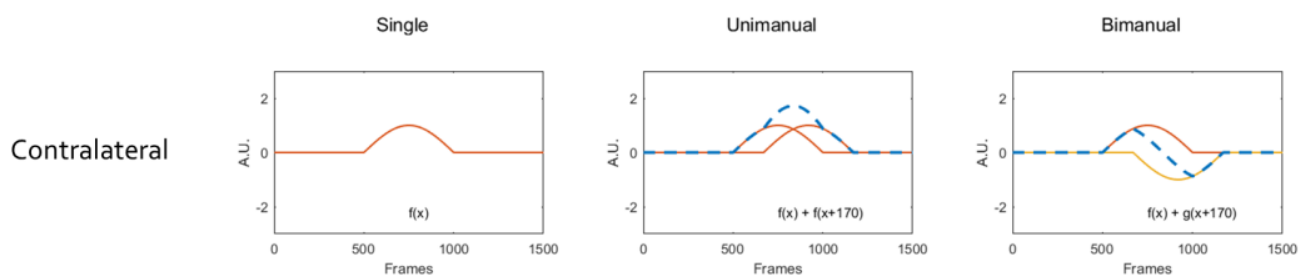

Ipsilateral
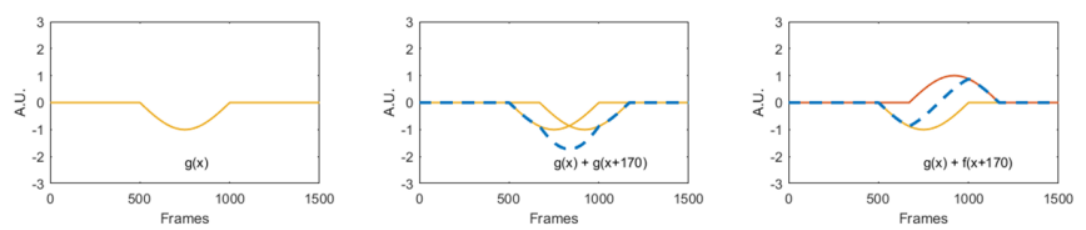

\section{b. Results}
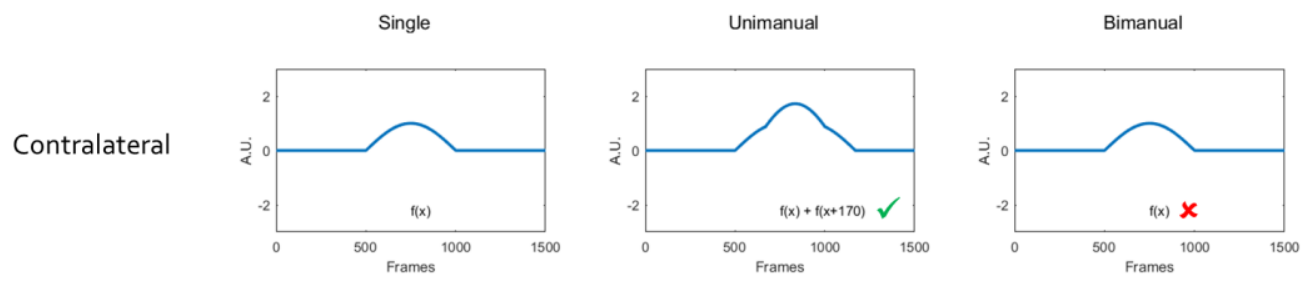

Ipsilateral
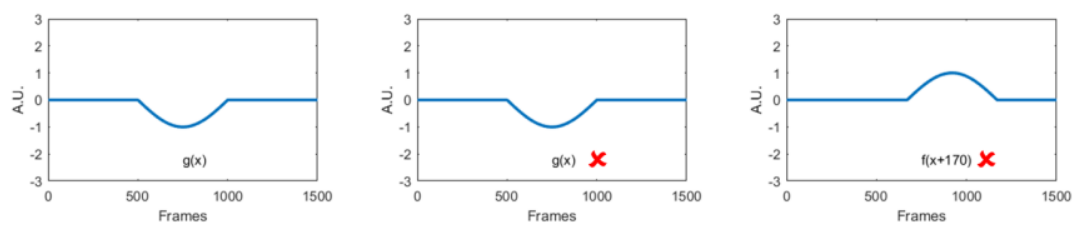

\section{c. Interpretation}

unimanual

bimanual

Key 1

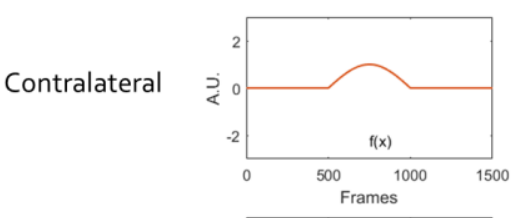

Ipsilateral

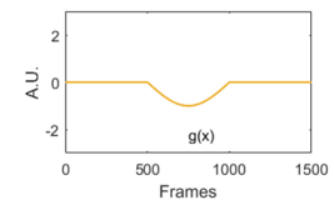

Key 2
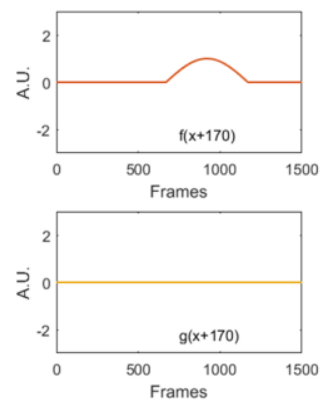

Key 1
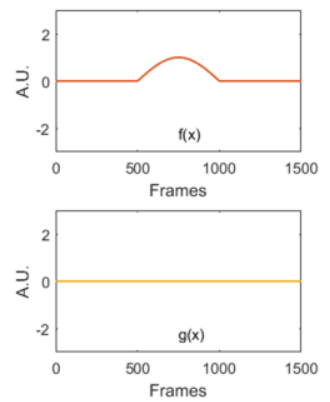

Key 2
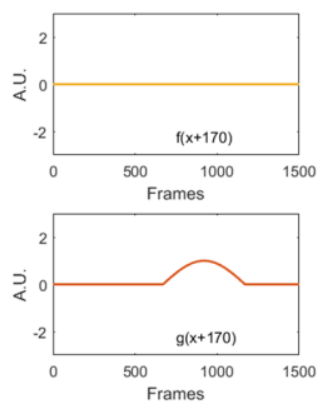

Figure 5. Illustration of (a) the additive hypothesis, (b) the observed patterns, and (c) their interpretation. (a) is similar to Figure 1. (b) Results are put in the form of equations, based on the computation from simple component time -courses (f[t] and $\mathrm{g}[\mathrm{t}]$ ) that lead to the appropriate amplitude and polarity. Only the contralateral electrode of unimanual sequences is in line with the additive hypothesis. (c) The "single" pattern that would be associated with each stroke (Key 1 and Key 2) is plotted separately for unimanual and bimanual sequences. Activity over the contralateral hemisphere to each keystroke is plotted in orange lines, and over the ipsilateral hemisphere in yellow lines. Contralateral and ipsilateral hemispheres are swapped for bimanual sequences between Key 1 and Key 2. 
Neurophysiological activity recorded from ipsilateral electrodes is typically linked, in choice RT tasks, with inhibition processes (Burle et al., 2004; Diedrichsen et al., 2013; Meckler et al., 2011; Servant et al., 2016). Both single keystrokes and unimanual sequences revealed an ipsilateral positive component, which, in accord with the literature, we interpret as inhibition (Burle et al., 2004; Vidal et al., 2015). Moreover, the ipsilateral components in both conditions were similar in terms of amplitude and timing. This similarity is incompatible with an additive hypothesis for the ipsilateral component (see Figure 5b). Specifically, there is no "doubling" of the ipsilateral component when there are two keystrokes using the same hand. Hence, we conclude that ipsilateral inhibition is independent of the number of keystrokes to be produced (one or two) in our experimental setting. This is in sharp contrast with the activation component that followed closely the number of keystrokes (Figure $5 \mathrm{c}$ ). The differences in whether activation and inhibition components associated with the two keystrokes summed or not supports the commonly held view that that activation and inhibition arise from independent processes (Meckler et al., 2011; Tandonnet, Burle, Vidal, \& Hasbroucq, 2003).

Turning to the bimanual sequences, we did not see any ipsilateral activity that could be linked to inhibition. We show that it could not have been hindered by activation components either, like the additive hypothesis would suggest (Figure 5a), otherwise we would have observed lower amplitude for the activation component. Moreover, the exact pattern predicted by the additive model has been found in the case of corrective actions, when one erroneous strike is followed by a ballistic correction (Rodriguez-Fornells, Schmitt, Kutas, \& Münte, 2002; Roger, Núñez Castellar, Pourtois, \& Fias, 2014), which demonstrates the validity of the additive hypothesis in a different context.

Figure 5c summarizes our findings. We depict the lack of an inhibitory component in the bimanual condition and the presence of an inhibition component in unimanual sequences associated with the first keystroke of the sequence only. This interpretation matches the timing of the inhibitory component relative to the first keystroke for unimanual sequences.

By definition, bimanual sequences require both effectors. Conversely, a single keystroke and a unimanual sequence are similar in that they both require the use of a unique effector. The present findings suggest that ipsilateral inhibition depends on the effectors recruited in a particular action (R. Q. Cui \& Deecke, 1999): if an effector is not required for a particular trial, e.g., as is the case for unimanual sequences, it gets "excluded" through inhibition. Crucially, we demonstrate here that inhibition does not depend on the number of keystrokes performed. This contradicts the idea that ipsilateral inhibition corresponds to inhibition of following items and suggests a different process at play than that postulated by CQ models (Houghton, 1990; Pinet et al., 2015).

The interpretation of ipsilateral positivity as the inhibition of an effector makes sense in the context of various models. First, it can be linked to evidence accumulation models in decision making, such as the Drift Diffusion Model (Ratcliff, Smith, Brown, \& McKoon, 2016), where evidence for an alternative is equally evidence against the other alternative (Servant et al., 2016; Wolpert \& Landy, 2012). Recent experimental evidence suggested that decision making arises from a continuous flow of information from the perceptual system to the motor system, leading to the build-up of evidence for one alternative along the system (e.g., Donner et al. 2009), which makes it plausible to find response competition up to the motor system (Calderon, Gevers, \& Verguts, 2018). Previous studies have described inhibition at the level of an effector, using TMS (Burle et al. 2002; Duque et al. 2010; Greenhouse et al. 2015; for typing see Behmer et al. 2018). Interestingly, inhibition seemed restricted to relevant effectors, i.e., those involved in the block/task (Labruna et al., 2014). Our findings are compatible with this idea and go further by showing that inhibition could be applied dynamically at the level of single trials within a block, alternating between trials where an effector had to be excluded or not. 
Second, and specifically relative to typing, our results are compatible with the features of keystroke representations postulated by cognitive models of this skill (Grudin, 1983; Rumelhart \& Norman, 1982). Keystroke representations or "schemata" assume a crucial role of the effector; they are thought to hold information about the hand and finger responsible for typing each keystroke, as well as the spatial position of the key on the keyboard. The validity of such features had been confirmed behaviorally (Logan, 2003). Our results provide the first neurophysiological evidence for such keystroke schemata, by showing that the effector (hand) is a relevant feature at the physiological level. Moreover, we go one step further by arguing that "hand" should be viewed as a specific decision selection level, a hypothesis that is still absent from current models of typing (Logan, 2018), although it is present in other models of action selection (Calderon et al., 2018; Herbort \& Rosenbaum, 2014). Indeed, for an effector to be excluded during response programming, there needs to be a processing step dealing with selecting the appropriate effector(s) for the current trial. We speculate that this step comes right after keystroke selection when keystroke features have been activated and unnecessary features (e.g., an irrelevant effector) can be excluded.

In a larger context, we set up this study to test assumptions of cognitive models of sequence production, namely successive activation and inhibition between items, by searching for their physiological implementation. Our results confirmed the additive activation over contralateral hemisphere(s) associated with each keystroke. However, the physiological evidence we report contradicts one prediction from CQ models, namely that each item inhibits the following ones. While we could not find traceable evidence for such inhibition between items, we report inhibition at the effector level. By demonstrating the existence of inhibition of irrelevant effectors, our results can then further constrain cognitive models. For a simple computation that could be "pressing two keys in the right order", we were able to describe some crucial properties of the neurophysiological implementation, based on previous assumptions, and to further clarify the algorithm that needs to be performed (Krakauer, Ghazanfar, Gomez-Marin, MacIver, \& Poeppel, 2017; Marr, 1982).

Contrasting sequences to single keystrokes leads to a final question regarding response complexity. Typing two keystrokes in a row is necessarily more complex than typing one, both in terms of planning and execution (Greenhouse et al. 2015). It is true that we observed higher amplitude for sequences compared to single keystrokes over midline electrodes, which would suggest stronger processing associated with the complexity of the response. This observation is spatially compatible with the activity of premotor areas (PMd and SMA) associated with response planning (recall that we report current source density estimates derived from spatial sharpening by Laplacian: Shima and Tanji 1998; Tanji 2001; Verwey et al. 2002; Vidal et al. 2003; Verstynen and Ivry 2011). However, RTs were not different between single keystrokes and sequences. While this result can seem surprising in light of previous findings on sequence production (Henry \& Rogers, 1960; Sternberg, Monsell, Knoll, \& Wright, 1978), specifics about our paradigm such as the high number of repetitions of each item, the short length of sequences ( 2 strokes maximum), as well as the fact that one- and two-keystroke conditions were presented in different blocks could all explain a performance near ceiling and no effect of response complexity. Moreover, the activation components of single keystrokes and bimanual sequences were similar; likewise, the inhibition component of single keystrokes and unimanual sequences were similar too. The fact that we can observe similar activity for single keystrokes and sequences shows that the activity we are observing over motor cortices is not due to response complexity, but rather specific to mechanisms of sequence production. Thus, we provided a refined description along the motor hierarchy, from planning to execution (Grafton \& Hamilton, 2007). Future studies should further characterize the specific role of each area along the motor 
pathway and their interactions particularly during sequence production, where typing provides a fruitful model, including upstream areas such as the prefrontal cortex or the posterior parietal cortex (Averbeck, Chafee, Crowe, \& Georgopoulos, 2003; Bohland \& Guenther, 2006; H. Cui \& Andersen, 2007; Koechlin \& Jubault, 2006; Le, Vesia, Yan, Niemeier, \& Crawford, 2014; Nakajima, Hosaka, Tsuda, Tanji, \& Mushiake, 2013).

We also note that our analysis of the lateralization pattern was driven by previous descriptions of positive and negative potentials that have been observed around the response time at relatively specific electrode locations. This approach is justified by previous founding work in motor control and typing (Pinet et al., 2015; Scaltritti et al., 2018; Vidal et al., 2003). Nonetheless, future broader explorations will have to reveal and explore other EEG components that may reflect response preparation in this task. This might be in particular relevant to characterize whether there is a mixture of processes in the programming of bimanual sequences (R. Q. Cui \& Deecke, 1999). In addition, we did not present analyses in the frequency domain. This approach has been useful in the past to characterize response preparation in complement to the analysis of potentials (de Jong et al., 2006; Gladwin et al., 2008; Park et al., 2013). While it did not fit with our detailed hypotheses regarding the link with CQ models, we believe further exploration should take advantage of frequency analyses to characterize sequence preparation (Pinet et al., 2016, 2015).

Typing is a complex sequential activity that requires precise hand and finger coordination. Using metrics that have proven to be effective in studying response selection in motor control, we demonstrated that contralateral activation was directly linked to response execution, in an additive fashion. The activity over ipsilateral cortex shed light on the intricacies of response programming. These results led us to argue for the existence of an effector processing level that is compatible with models of both decision making and typing. In that sense, our data help to bridge the gap between cognitive models of sequence production and their physiological implementation.

\section{Acknowledgements}

Research supported by the Ministère de l'Enseignement Supérieur et de la Recherche, by the European Research Council under the European Community's Seventh Framework Program (FP7/2007- 2013; grant agreement $n^{\circ}$ 263575), by grants ANR-16-CONV-0002 (ILCB), ANR-11-LABX-0036 (BLRI) of the French Agence Nationale de la Recherche, and by the Excellence Initiative of Aix-Marseille University (A*MIDEX).

\section{References}

Alegre, M., De Gurtubay, I. G., Labarga, A., Iriarte, J., Malanda, A., \& Artieda, J. (2004). Alpha and beta oscillatory activity during a sequence of two movements. Clinical Neurophysiology, 115, 124-130. https://doi.org/10.1016/S13882457(03)00311-0

Averbeck, B. B., Chafee, M. V, Crowe, D. A., \& Georgopoulos, A. P. (2002). Parallel processing of serial movements in prefrontal cortex. Proceedings of the National Academy of Sciences of the United States of America, 99(20), 1317213177. https://doi.org/10.1073/pnas.162485599

Averbeck, B. B., Chafee, M. V, Crowe, D. A., \& Georgopoulos, A. P. (2003). Neural activity in prefrontal cortex during copying geometrical shapes. I. Single cells encode shape, sequence, and metric parameters. Experimental Brain Research. Experimentelle Hirnforschung. Experimentation Cerebrale, 150(2), 127-141. https://doi.org/10.1007/s00221-003-1416-6

Baayen, R. H., Davidson, D. J., \& Bates, D. M. (2008). Mixed-effects modeling with crossed random effects for subjects and 
items. Journal of Memory and Language, 59(4), 390-412. https://doi.org/10.1016/j.jml.2007.12.005

Bates, D. M., Mächler, M., Bolker, B., \& Walker, S. (2015). Fitting Linear Mixed-Effects Models Using 1me4. Journal of Statistical Software, 67(1). https://doi.org/10.18637/jss.v067.i01

Behmer, L. P., Jantzen, K. J., Martinez, S., Walls, R., Amir-Brownstein, E., Jaye, A., ... Crump, M. J. C. (2018). Parallel regulation of past, present, and future actions during sequencing. Journal of Experimental Psychology: Human Perception and Performance, 44(8), 1147-1152. https://doi.org/10.1037/xhp0000507

Bohland, J. W., \& Guenther, F. H. (2006). An fMRI investigation of syllable sequence production. NeuroImage, 32(2), 821841. https://doi.org/10.1016/j.neuroimage.2006.04.173

Burle, B., Bonnet, M., Vidal, F., Possamaï, C.-A., \& Hasbroucq, T. (2002). A transcranial magnetic stimulation study of information processing in the motor cortex: relationship between the silent period and the reaction time delay. Psychophysiology, 39(2), 207-217. https://doi.org/10.1017/S0048577202010077

Burle, B., Vidal, F., Tandonnet, C., \& Hasbroucq, T. (2004). Physiological evidence for response inhibition in choice reaction time tasks. Brain and Cognition, 56(2), 153-164. https://doi.org/10.1016/j.bandc.2004.06.004

Calderon, C. B., Gevers, W., \& Verguts, T. (2018). The unfolding action model of initiation times, movement times, and movement paths. Psychological Review, 125(5), 785-805. https://doi.org/10.1037/rev0000110

Coles, M. G. H. (1989). Modern mind-brain reading: Psychophysiology, physiology, and cognition. Psychophysiology, 26(3), 251-269. https://doi.org/10.1111/j.1469-8986.1989.tb01916.x

Cui, H., \& Andersen, R. A. (2007). Posterior Parietal Cortex Encodes Autonomously Selected Motor Plans. Neuron, 56(3), 552-559. https://doi.org/10.1016/j.neuron.2007.09.031

Cui, R. Q., \& Deecke, L. (1999). High resolution DC-EEG analysis of the Bereitschaftspotential and post movement onset potentials accompanying uni- or bilateral voluntary finger movements. Brain Topography, 11(3), 233-249. https://doi.org/10.1023/A:1022237929908

de Jong, R., Gladwin, T. E., \& M't Hart, B. (2006). Movement-related EEG indices of preparation in task switching and motor control. Brain Research, 1105(1), 73-82. https://doi.org/10.1016/j.brainres.2006.03.030

de Jong, R., Wierda, M., Mulder, G., \& Mulder, L. J. (1988). Use of partial stimulus information in response processing. Journal of Experimental Psychology. Human Perception and Performance, 14(4), 682-692. Retrieved from http://www.ncbi.nlm.nih.gov/pubmed/2974876

Delorme, A., \& Makeig, S. (2004). EEGLAB: An open source toolbox for analysis of single-trial EEG dynamics including independent component analysis. Journal of Neuroscience Methods, 134(1), 9-21. https://doi.org/10.1016/j.jneumeth.2003.10.009

Diedrichsen, J., Wiestler, T., \& Krakauer, J. W. (2013). Two distinct ipsilateral cortical representations for individuated finger movements. Cerebral Cortex, 23(6), 1362-1377. https://doi.org/10.1093/cercor/bhs120

Donner, T. H., Siegel, M., Fries, P., \& Engel, A. K. (2009). Buildup of choice-predictive activity in human motor cortex during perceptual decision making. Current Biology, 19(18), 1581-1585. https://doi.org/10.1016/j.cub.2009.07.066

Duque, J., Lew, D., Mazzocchio, R., Olivier, E., \& Ivry, R. B. (2010). Evidence for two concurrent inhibitory mechanisms during response preparation. The Journal of Neuroscience, 30(10), 3793-3802. https://doi.org/10.1523/JNEUROSCI.5722-09.2010

Fabiani, M., Gratton, G., Karis, D., \& Donchin, E. (1987). Definition, identification and reliability of the P300 component of the event related brain potential. Advances in Psychophysiology, 2(April), 1-78.

Gladwin, T. E., 't Hart, B. M., \& de Jong, R. (2008). Dissociations between motor-related EEG measures in a cued movement sequence task. Cortex, 44(5), 521-536. https://doi.org/10.1016/j.cortex.2007.10.005

Grafton, S. T., \& Hamilton, A. (2007). Evidence for a distributed hierarchy of action representation in the brain. Human Movement Science, 26(4), 590-616. https://doi.org/10.1016/j.micinf.2011.07.011.Innate

Gratton, G., Coles, M. G. H., Sirevaag, E. J., Eriksen, C. W., \& Donchin, E. (1988). Pre- and poststimulus activation of response channels: a psychophysiological analysis. Journal of Experimental Psychology. Human Perception and Performance, 14(3), 331-344.

Greenhouse, I., Saks, D., Hoang, T., \& Ivry, R. B. (2015). Inhibition during response preparation is sensitive to response complexity. Journal of Neurophysiology, 113(7), 2792-2800. https://doi.org/10.1152/jn.00999.2014

Greenhouse, I., Sias, A., Labruna, L., \& Ivry, R. B. (2015). Nonspecific Inhibition of the Motor System during Response Preparation. Journal of Neuroscience, 35(30), 10675-10684. https://doi.org/10.1523/JNEUROSCI.1436-15.2015

Grudin, J. (1983). Error Patterns in Novice and Skilled Transcription Typing. In Cognitive aspects of skilled typewriting (pp. 121-143).

Henry, F. M., \& Rogers, D. E. (1960). Increased response latency for complicated movements and a "memory drum" theory of neuromotor reaction. Research Quarterly. American Association for Health, Physical Education and Recreation, $31(3), 448-458$

Herbort, O., \& Rosenbaum, D. A. (2014). What is chosen first, the hand used for reaching or the target that is reached? Psychonomic Bulletin \& Review, 21(1), 170-177. https://doi.org/10.3758/s13423-013-0488-y

Houghton, G. (1990). The problem of serial order: A neural network model of sequence learning and recall. In Current 
research in natural language generation (pp. 287-319). London: Academic Press.

Jaeger, T. F. (2008). Categorical data analysis: Away from ANOVAs (transformation or not) and towards logit mixed models. Journal of Memory and Language, 59(4), 434-446. https://doi.org/10.1016/j.jml.2007.11.007

Koechlin, E., \& Jubault, T. (2006). Broca's Area and the Hierarchical Organization of Human Behavior. Neuron, 50(6), 963974. https://doi.org/10.1016/j.neuron.2006.05.017

Krakauer, J. W., Ghazanfar, A. A., Gomez-Marin, A., MacIver, M. A., \& Poeppel, D. (2017). Neuroscience Needs Behavior: Correcting a Reductionist Bias. Neuron, 93(3), 480-490. https://doi.org/10.1016/j.neuron.2016.12.041

Labruna, L., Lebon, F., Duque, J., Klein, P.-A., Cazares, C., \& Ivry, R. B. (2014). Generic inhibition of the selected movement and constrained inhibition of nonselected movements during response preparation. Journal of Cognitive Neuroscience, 26(2), 269-278. https://doi.org/10.1162/jocn_a_00492

Lashley, K. S. (1951). The Problem of Serial Order in Behavior.

Le, A., Vesia, M., Yan, X., Niemeier, M., \& Crawford, J. D. (2014). The right anterior intraparietal sulcus is critical for bimanual grasping: A TMS study. Cerebral Cortex, 24(10), 2591-2603. https://doi.org/10.1093/cercor/bht115

Logan, G. D. (2003). Simon-Type Effects: Chronometric Evidence for Keypress Schemata in Typewriting. Journal of Experimental Psychology. Human Perception and Performance, 29(4), 741-757. https://doi.org/10.1037/00961523.29.4.741

Logan, G. D. (2018). Automatic control: How experts act without thinking. Psychological Review, 125(4), 453-485. https://doi.org/10.1037/rev0000100

Logan, G. D., Miller, A. E., \& Strayer, D. L. (2011). Electrophysiological evidence for parallel response selection in skilled typists. Psychological Science, 22(1), 54-56. https://doi.org/10.1177/0956797610390382

Maris, E., \& Oostenveld, R. (2007). Nonparametric statistical testing of EEG- and MEG-data. Journal of Neuroscience Methods, 164, 177-190. https://doi.org/10.1016/j.jneumeth.2007.03.024

Marr, D. (1982). Vision: A computational approach. San Francisco: Freeman \& Co.

Meckler, C., Allain, S., Carbonnell, L., Hasbroucq, T., Burle, B., \& Vidal, F. (2011). Executive control and response expectancy: a Laplacian ERP study. Psychophysiology, 48(3), 303-311. https://doi.org/10.1111/j.14698986.2010.01077.x

Meyer, D. E., Osman, A. M., Irwin, D. E., \& Yantis, S. (1988). Modern mental chronometry. Biological Psychology, 26, 367.

Meynier, C., Burle, B., Possamaï, C.-A., \& Vidal, F. (2009). Neural inhibition and interhemispheric connections in twochoice reaction time: A Laplacian ERP study. Psychophysiology, 46(4), 726-730. https://doi.org/10.1111/j.14698986.2009.00818.x

Nakajima, T., Hosaka, R., Tsuda, I., Tanji, J., \& Mushiake, H. (2013). Two-Dimensional Representation of Action and ArmUse Sequences in the Presupplementary and Supplementary Motor Areas. Journal of Neuroscience, 33(39), 1553315544. https://doi.org/10.1523/JNEUROSCI.0855-13.2013

Oostenveld, R., Fries, P., Maris, E., \& Schoffelen, J. M. (2011). FieldTrip: Open source software for advanced analysis of MEG, EEG, and invasive electrophysiological data. Computational Intelligence and Neuroscience, 2011. https://doi.org/10.1155/2011/156869

Park, H., Kim, J. S., \& Chung, C. K. (2013). Differential Beta-Band Event-Related Desynchronization during Categorical Action Sequence Planning. PLoS ONE, 8(3). https://doi.org/10.1371/journal.pone.0059544

Perrin, F., Pernier, J., Bertrand, O., \& Echallier, J. F. (1989). Spherical splines for scalp potential and current density mapping. Electroencephalography and Clinical Neurophysiology, 72(2), 184-187.

Pinet, S., Dubarry, A.-S., \& Alario, F.-X. (2016). Response retrieval and motor planning during typing. Brain and Language, 159(1), 74-83. https://doi.org/10.1016/j.bandl.2016.05.012

Pinet, S., Hamamé, C. M., Longcamp, M., Vidal, F., \& Alario, F.-X. (2015). Response planning in word typing: Evidence for inhibition. Psychophysiology, 52(4), 524-531. https://doi.org/10.1111/psyp.12373

Ratcliff, R., Smith, P. L., Brown, S. D., \& McKoon, G. (2016). Diffusion Decision Model: Current Issues and History. Trends in Cognitive Sciences, 20(4), 260-281. https://doi.org/10.1016/j.tics.2016.01.007

Rodriguez-Fornells, A., Schmitt, B. M., Kutas, M., \& Münte, T. F. (2002). Electrophysiological estimates of the time course of semantic and phonological encoding during listening and naming. Neuropsychologia, 40(7), 778-787.

Roger, C., Núñez Castellar, E., Pourtois, G., \& Fias, W. (2014). Changing your mind before it is too late: The electrophysiological correlates of online error correction during response selection. Psychophysiology, 51(8), 746-760. https://doi.org/10.1111/psyp.12224

Rumelhart, D. E., \& Norman, D. A. (1982). Simulating a Skilled Typist : A Study of Skilled Cognitive-Motor Performance. Cognitive Science, 6, 1-36.

Scaltritti, M., Alario, F.-X., \& Longcamp, M. (2018). The scope of planning in serial order actions: The case of typing. Journal of Cognitive Neuroscience, 1-10.

Scaltritti, M., Pinet, S., Longcamp, M., \& Alario, F.-X. (2017). On the functional relationship between language and motor processing in typewriting: an EEG study. Language, Cognition and Neuroscience, 0(0), 1-16. 
Keystroke sequences at the cortical level

https://doi.org/10.1080/23273798.2017.1283427

Selen, L. P. J., Shadlen, M. N., \& Wolpert, D. M. (2012). Deliberation in the Motor System: Reflex Gains Track Evolving Evidence Leading to a Decision. Journal of Neuroscience, 32(7), 2276-2286. https://doi.org/10.1523/JNEUROSCI.5273-11.2012

Servant, M., White, C., Montagnini, A., \& Burle, B. (2016). Linking Theoretical Decision-making Mechanisms in the Simon Task with Electrophysiological Data: A Model-based Neuroscience Study in Humans. Journal of Cognitive Neuroscience, 28(10), 1501-1521. https://doi.org/10.1162/jocn_a_00989

Shima, K., \& Tanji, J. (1998). Both supplementary and presupplementary motor areas are crucial for the temporal organization of multiple movements. Journal of Neurophysiology, 80(6), 3247-3260. https://doi.org/80:3247-3260

Sternberg, S., Monsell, S., Knoll, R. L., \& Wright, C. E. (1978). The latency and duration of rapid movement sequences: comparisons of speech and typewriting. In Information Processing in Motor Control and Learning.

Tadel, F., Baillet, S., Mosher, J. C., Pantazis, D., \& Leahy, R. M. (2011). Brainstorm: a user-friendly application for MEG/EEG analysis. Computational Intelligence and Neuroscience, $2011,879716$. https://doi.org/10.1155/2011/879716

Tandonnet, C., Burle, B., Vidal, F., \& Hasbroucq, T. (2003). The influence of time preparation on motor processes assessed by surface Laplacian estimation. Clinical Neurophysiology, 114, 2376-2384. https://doi.org/10.1016/S13882457(03)00253-0

Tanji, J. (2001). Sequential organization of multiple movements: involvement of cortical motor areas. Annual Review of Neuroscience, 24(1), 631-651. https://doi.org/10.1146/annurev.neuro.24.1.631

Verstynen, T., \& Ivry, R. B. (2011). Network dynamics mediating ipsilateral motor cortex activity during unimanual actions. Journal of Cognitive Neuroscience, 23(9), 2468-2480. https://doi.org/10.1162/jocn.2011.21612

Verwey, W. B., Lammens, R., \& van Honk, J. (2002). On the role of the SMA in the discrete sequence production task: a TMS study. Transcranial Magnetic Stimulation. Neuropsychologia, 40, 1268-1276. https://doi.org/10.1016/S00283932(01)00221-4

Vidal, F., Burle, B., Spieser, L., Carbonnell, L., Meckler, C., Casini, L., \& Hasbroucq, T. (2015). Linking EEG signals, brain functions and mental operations: Advantages of the Laplacian transformation. International Journal of Psychophysiology, 97(3), 221-232. https://doi.org/10.1016/j.ijpsycho.2015.04.022

Vidal, F., Grapperon, J., \& Bonnet, M. (2003). The nature of unilateral motor commands in between- hand choice tasks as revealed by surface Laplacian estimation. Psychophysiology, 40, 796-805.

Wolpert, D. M., \& Landy, M. S. (2012). Motor control is decision-making. Current Opinion in Neurobiology, 22(6), 9961003. https://doi.org/10.1016/j.conb.2012.05.003 